\title{
TITLE:
}

\section{Hawking-Moss instanton in nonlinear massive gravity}

$\operatorname{AUTHOR}(S)$ :

Zhang, Ying-li; Saito, Ryo; Sasaki, Misao

\section{CITATION:}

Zhang, Ying-li ... [et al]. Hawking-Moss instanton in nonlinear massive gravity. Journal of Cosmology and Astroparticle Physics 2013, 2013(02): 029.

\section{ISSUE DATE:}

2013-02-01

URL:

http://hdl.handle.net/2433/173708

\section{RIGHT:}

(C) 2013 IOP Publishing Ltd and Sissa Medialab srl; この論文は出版社版 でありません。引用の際には出版社版をご確認ご利用ください。;This is not the published version. Please cite only the published version. 


\title{
Hawking-Moss instanton in nonlinear massive gravity
}

\author{
Ying-li Zhang*, Ryo Saito ${ }^{\dagger}$ and Misao Sasaki ${ }^{\ddagger}$ \\ Yukawa Institute for Theoretical Physics, Kyoto University, Kyoto 606-8502, Japan
}

(Dated: February 21, 2013)

\begin{abstract}
As a first step toward understanding a lanscape of vacua in a theory of non-linear massive gravity, we consider a landscape of a single scalar field and study tunneling between a pair of adjacent vacua. We study the Hawking-Moss (HM) instanton that sits at a local maximum of the potential, and evaluate the dependence of the tunneling rate on the parameters of the theory. It is found that provided with the same physical HM Hubble parameter $H_{H M}$, depending on the values of parameters $\alpha_{3}$ and $\alpha_{4}$ in the action (2.2), the corresponding tunneling rate can be either enhanced or suppressed when compared to the one in the context of General Relativity (GR). Furthermore, we find the constraint on the ratio of the physical Hubble parameter to the fiducial one, which constrains the form of potential. This result is in sharp contrast to GR where there is no bound on the minimum value of the potential.
\end{abstract}

\section{INTRODUCTION}

It is of great interest to promote Einstein's gravity theory to a massive one [1]. However, it has been shown that Lorentz-invariant massive gravity theories would suffer from the Boulware-Deser (BD) ghost instability [2, 3] (For a review, see e.g. Ref. [4, 5]). Recently, a non-linear construction of a Lorentz-invariant massive gravity theory has been proposed in Refs. 6- 8], where the nonlinear terms are designed so that the BD ghost is removed by using the constraint equations [9, 10]. Many researches have been made to explore its physical consequences since this breakthrough.

One of the most interesting results among these explorations is that such a massive gravity theory allows self-accelerating solutions (e.g. for Minkowski fiducial metric, see 11 16]; for deSitter fiducial metric, see [17]; for a more general case where fiducial metric respects only isotropy, see [18]). While cosmological solutions in usual massive gravity theories are known to suffer from appearance of a ghost within 5 degrees of freedom of the massive graviton [19 21], it has been proved that perturbations on the solution found in Ref. [12] do not have massive degrees of freedom and evade the ghost instability at linear order [22]. ${ }^{1}$

Though the mass of graviton may successfully explain the current acceleration, it is still to be explained why the cosmological constant is so small while large quantum corrections are expected. ${ }^{2}$ In spite of many attempts, this problem, so called "the cosmological constant problem" 26, 27], has not been solved yet.

A possible resolution to this problem is the anthropic selection of the cosmological constant in the landscape of vacua [26 28]. In the landscape of vacua, a vacuum can be unstable with respect to tunneling to other vacua. The main interest of this paper is to investigate how the stability of a vacuum is determined in the non-linear massive gravity theory. We investigate how the tunneling rate in general relativity (GR) is modified by the graviton mass terms and clarify dependence on the model parameters. Moreover, an interesting possibility will arise if the graviton mass depends on a value of a scalar field [29 31], e.g., the tunneling field. In this case, the effective cosmological constant of a vacuum can be larger than the other while its potential energy is smaller because of the contributions from the mass terms. It will be interesting to see how the tunneling process proceeds in this case.

In this paper, we consider the Hawking-Moss (HM) solution [32] for a scalar field with minimal coupling to gravity to understand the tunneling process in non-linear massive gravity. We set up the model and found a bounce solution corresponding to a HM solution. Based on this solution, we evaluate the HM action and the contributions from the graviton mass terms. We find they may either enhance or suppress the tunneling rate compared to that in GR.

This paper is organized as follows. In Sec. III we setup the Lagrangian for our model. In Sec. III we formulate the equations of motion (EOM) and solve the constraint equation. In Sec. IV the HM solution is

\footnotetext{
* E-mail address: yingli@yukawa.kyoto-u.ac.jp

$\dagger$ E-mail address: rsaito@yukawa.kyoto-u.ac.jp

¥ E-mail address: misao@yukawa.kyoto-u.ac.jp

1 See also the recent discussion on the ghost instability at nonlinear level [23 25].

${ }^{2}$ Note that the graviton mass does not receive a large quantum correction because the other parts, where the general coordinate invariance symmetry is respected, do not induce a correction to the mass terms.
} 


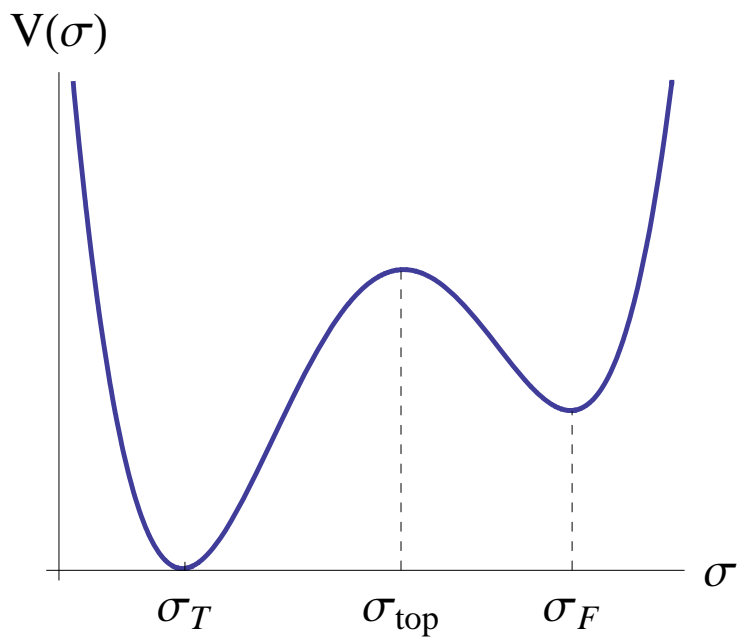

FIG. 1: Illustration of the potential $V(\sigma)$ with two local minima $\sigma_{F}$ and $\sigma_{T}$, which correspond to the false and true vacuum respectively. Here the $\sigma_{\text {top }}$ labels its local maximum value.

studied. To analyze the effect of the graviton mass terms in details, we consider several specific combinations of the parameters.

Throughout the paper, the Lorentzian metric signature is set to be $(-,+,+,+)$.

\section{SETUP}

We study a tunneling of a minimally coupled scalar field $\sigma$ between two vacua in non-linear massive gravity. It is described by a 4-dimensional metric $g_{\mu \nu}$, a fiducial metric $G_{a b}$, and the Stückelberg fields $\phi^{a} .{ }^{3}$ The action is given by ${ }^{4}$

$$
\begin{aligned}
S & =I_{g}+I_{m}, \\
I_{g} & \equiv \int \mathrm{d}^{4} x \sqrt{-g}\left[\frac{R}{2}+m_{g}^{2}\left(\mathcal{L}_{2}+\alpha_{3} \mathcal{L}_{3}+\alpha_{4} \mathcal{L}_{4}\right)\right], \\
I_{m} & \equiv-\int \mathrm{d}^{4} x \sqrt{-g}\left[\frac{1}{2}(\partial \sigma)^{2}+V(\sigma)\right],
\end{aligned}
$$

where

$$
\begin{aligned}
& \mathcal{L}_{2}=\frac{1}{2}\left([\mathcal{K}]^{2}-\left[\mathcal{K}^{2}\right]\right), \\
& \mathcal{L}_{3}=\frac{1}{6}\left([\mathcal{K}]^{3}-3[\mathcal{K}]\left[\mathcal{K}^{2}\right]+2\left[\mathcal{K}^{3}\right]\right), \\
& \mathcal{L}_{4}=\frac{1}{24}\left([\mathcal{K}]^{4}-6[\mathcal{K}]^{2}\left[\mathcal{K}^{2}\right]+3\left[\mathcal{K}^{2}\right]^{2}+8[\mathcal{K}]\left[\mathcal{K}^{3}\right]-6\left[\mathcal{K}^{4}\right]\right),
\end{aligned}
$$

and

$$
\mathcal{K}_{\nu}^{\mu} \equiv \delta_{\nu}^{\mu}-\sqrt{g^{\mu \sigma} G_{a b}(\phi) \partial_{\nu} \phi^{a} \partial_{\sigma} \phi^{b}} .
$$

In this paper we assume $G_{a b}$ is non-dynamical as discussed below. The potential $V(\sigma)$ is assumed to have two local minima $\sigma_{F}$ and $\sigma_{T}$, where the former corresponds to the false vacuum, and a local maximum between them, $\sigma=\sigma_{\text {top }}$ (see Fig. 1).

3 We use Greek letters $\mu, \nu, \ldots$ for the spacetime indices and the Latin letters $i, j, \ldots$ for the space indices, while the Latin indices $a, b, \ldots$ for the internal space (Lorentz frame) indices. Repeated indices are understood to be summed over unless otherwise stated.

${ }^{4}$ We use the natural units throughout this paper. 
Provided the action (2.1), the Euclidean action can be obtained by $S_{E}=i S\left[x^{0} \rightarrow i x_{E}^{0}\right]$. Then, in the semiclassical limit, the tunneling rate per unit time per unit volume is given in terms of the Euclidean action as

$$
\Gamma / V=A e^{-B} ; \quad B=S_{E}\left[\bar{g}_{\mu \nu, B}, \bar{\phi}_{B}\right]-S_{E}\left[\bar{g}_{\mu \nu, F}, \bar{\phi}_{F}\right],
$$

where $\left\{\bar{g}_{\mu \nu, B}, \bar{\phi}_{B}\right\}$ is the so-called bounce solution, or an instanton, a solution of the Euclidean equations of motion with appropriate boundary conditions, and $\left\{\bar{g}_{\mu \nu, F}, \bar{\phi}_{F}\right\}$ is the solution staying at the false vacuum [33]. Among the bounce solutions, the one that gives the least action dominates the tunneling.

Conventionally a bounce solution $\left\{\bar{g}_{\mu \nu, B}, \bar{\phi}_{B}\right\}$ is explored assuming $O(4)$-symmetry. This is because it was proved that an $O(4)$-symmetric solution gives the lowest action for a wide class of scalar-field theories 35$]$. It is therefore reasonable to assume the same even in the presence of gravity [33]. Hence the metric would take the form,

$$
g_{\mu \nu} \mathrm{d} x^{\mu} \mathrm{d} x^{\nu}=N(\xi)^{2} \mathrm{~d} \xi^{2}+a(\xi)^{2} \Omega_{i j} \mathrm{~d} x^{i} \mathrm{~d} x^{j},
$$

where the second term $\Omega_{i j} \mathrm{~d} x^{i} \mathrm{~d} x^{j}$ is the metric on a three-sphere $(K>0)$,

$$
\Omega_{i j} \equiv \delta_{i j}+\frac{K \delta_{i l} \delta_{j m} x^{l} x^{m}}{1-K \delta_{l m} x^{l} x^{m}} .
$$

However, in massive gravity, this may not be always true if the fiducial metric $G_{a b}$ does not respect the symmetry.

In this paper, to avoid possible complications due to the nature of the fiducial metric, we assume that it is given by the de Sitter metric with the "Hubble parameter" F, namely,

$$
G_{a b}(\phi) \mathrm{d} \phi^{a} \mathrm{~d} \phi^{b} \equiv-\left(\mathrm{d} \phi^{0}\right)^{2}+b\left(\phi^{0}\right)^{2} \Omega_{i j} \mathrm{~d} \phi^{i} \mathrm{~d} \phi^{j},
$$

where

$$
b\left(\phi^{0}\right) \equiv F^{-1} \sqrt{K} \cosh \left(F \phi^{0}\right) .
$$

Then an $O(4)$-symmetric solution can be obtained by setting

$$
\phi^{0}=f(\xi), \quad \phi^{i}=x^{i} .
$$

The absence of the BD ghost has been proved for a general fiducial metric in 10] and the de Sitter fiducial metric has been recently investigated in [17]. In contrast to the Minkowski fiducial metric, the de Sitter metric is shown to lead to a wider range of cosmological solutions [17].

At this level, the origin of the fiducial metric is unclear: It may be a non-dynamical metric of the Stückelberg field space or one of the two dynamical metrics in bimetric gravity [36 38]. In the later case, it may be natural to use the Euclidean signature also for the fiducial metric, which can be accomplished by making the replacement, $\phi^{0} \rightarrow i \phi_{\mathrm{E}}^{0}\left(f \rightarrow i f_{E}\right)$, in Eq. (2.11). In this case, the $O(4)$-symmetric solutions would exist also in the case that the fiducial metric is given by the Minkowski metric and the bounce solution could be found in a similar way by setting $b\left(\phi^{0}\right) \equiv \sqrt{K} \phi^{0}$.

Here, we stick to the Lorentzian signature for the fiducial metric because it is non-dynamical in our case. Nevertheless, thanks to the assumption of the de Sitter fiducial metric, we may adopt the $O(4)$-ansatz.

\section{BASIC EQUATIONS}

Here we derive basic equations. First we write down the Euclidean version of the action (2.1). Inserting the $O(4)$-ansatz (2.7) and (2.11) into the Euclidean version of (2.5), we find

$$
\mathcal{K}_{0}^{0}=1-\frac{\sqrt{-\dot{f}^{2}}}{N}, \quad \mathcal{K}_{j}^{i}=\left(1-\frac{b}{a}\right) \delta_{j}^{i}, \quad \mathcal{K}_{0}^{i}=0, \quad \mathcal{K}_{i}^{0}=0,
$$


where the dot means a derivative with respect to the radial coordinate, ${ }^{\cdot} \equiv \mathrm{d} / \mathrm{d} \xi$. This gives

$$
\begin{array}{r}
{[\mathcal{K}]=\left(1-\frac{\sqrt{-\dot{f}^{2}}}{N}\right)+3\left(1-\frac{b}{a}\right),} \\
{\left[\mathcal{K}^{2}\right]=\left(1-\frac{\sqrt{-\dot{f}^{2}}}{N}\right)^{2}+3\left(1-\frac{b}{a}\right)^{2},} \\
{\left[\mathcal{K}^{3}\right]=\left(1-\frac{\sqrt{-\dot{f}^{2}}}{N}\right)^{3}+3\left(1-\frac{b}{a}\right)^{3},} \\
{\left[\mathcal{K}^{4}\right]=\left(1-\frac{\sqrt{-\dot{f}^{2}}}{N}\right)^{4}+3\left(1-\frac{b}{a}\right)^{4} .}
\end{array}
$$

Then the gravity action is reduced to

$$
I_{g E}=\int \mathrm{d}^{4} x_{E} \sqrt{\Omega}\left[-3 K N a-\frac{3 \dot{a}^{2} a}{N}-m_{g}^{2}\left(L_{2 E}+\alpha_{3} L_{3 E}+\alpha_{4} L_{4 E}\right)\right],
$$

where

$$
\begin{aligned}
& \mathcal{L}_{2 E}=3 a(a-b)\left(2 N a-\sqrt{-\dot{f}^{2}} a-N b\right), \\
& \mathcal{L}_{3 E}=(a-b)^{2}\left(4 N a-3 \sqrt{-\dot{f}^{2}} a-N b\right), \\
& \mathcal{L}_{4 E}=(a-b)^{3}\left(N-\sqrt{-\dot{f}^{2}}\right),
\end{aligned}
$$

and the action for the tunneling field is reduced to

$$
I_{m E}=\int \mathrm{d}^{4} x a^{3} \sqrt{\Omega}\left[\frac{1}{2 N} \dot{\sigma}^{2}+N V(\sigma)\right],
$$

which coincides with the Euclidean version of the action (2.8) in Ref. [12] if we replace $f$ by $b(f)$.

\section{A. Constraint equation from the Stückelberg field}

The introduction of the Stückelberg field gives rise to an additional constrait equation. Variation of the action (3.6) with respect to $f$ gives

$$
\left(i \dot{a}+N b_{, f}\right)\left[\left(3-\frac{2 b}{a}\right)+\alpha_{3}\left(1-\frac{b}{a}\right)\left(3-\frac{b}{a}\right)+\alpha_{4}\left(1-\frac{b}{a}\right)^{2}\right]=0,
$$

where

$$
b_{, f} \equiv \frac{d b}{d f}=\sqrt{K} \sinh (F f)
$$

Note that the derivation of the above equation does not depends on the choice of a branch of $\sqrt{-\dot{f}^{2}}$.

Solving Eq. (3.9), we obtain two branches:

- Branch I

$$
N b_{, f}=-i \dot{a} .
$$


- Branch II

$$
\left(3-\frac{2 b}{a}\right)+\alpha_{3}\left(1-\frac{b}{a}\right)\left(3-\frac{b}{a}\right)+\alpha_{4}\left(1-\frac{b}{a}\right)^{2}=0 .
$$

The solution to this equation is given by

$$
b=X_{ \pm} a, \quad X_{ \pm} \equiv \frac{1+2 \alpha_{3}+\alpha_{4} \pm \sqrt{1+\alpha_{3}+\alpha_{3}^{2}-\alpha_{4}}}{\alpha_{3}+\alpha_{4}} .
$$

Hereafter, we call the choice $X_{+}$as Branch $\mathrm{II}_{+}$and $X_{-}$as Branch $\mathrm{II}_{-}$. In Appendix $\mathrm{A}$, it is shown that Branch I is equivalent to Branch II with a different set of parameters in the case of the HM solution. Hence, we concentrate on the Branch II solutions hereafter.

\section{B. Euclidean equations of motion}

Variation of the action (3.6) and (3.8) with respect to $N$ gives

$$
3\left(\frac{\dot{a}}{N a}\right)^{2}-\frac{3 K}{a^{2}}=\frac{1}{2 N^{2}} \dot{\sigma}^{2}-V(\sigma)-\rho_{g},
$$

where

$$
\rho_{g} \equiv-m_{g}^{2}\left(1-\frac{b}{a}\right)\left[3\left(2-\frac{b}{a}\right)+\alpha_{3}\left(1-\frac{b}{a}\right)\left(4-\frac{b}{a}\right)+\alpha_{4}\left(1-\frac{b}{a}\right)^{2}\right] .
$$

Inserting the solution (3.13) into the above, $\rho_{g}$ reduces to a cosmological constant,

$$
\rho_{g}\left(b / a=X_{ \pm}\right)=\Lambda_{ \pm} \equiv-\frac{m_{g}^{2}}{\left(\alpha_{3}+\alpha_{4}\right)^{2}}\left[\left(1+\alpha_{3}\right)\left(2+\alpha_{3}+2 \alpha_{3}^{2}-3 \alpha_{4}\right) \pm 2\left(1+\alpha_{3}+\alpha_{3}^{2}-\alpha_{4}\right)^{3 / 2}\right] .
$$

Variation of the action (3.8) with respect to $\sigma$ gives

$$
\frac{1}{N a^{3}}\left(\frac{a^{3} \dot{\sigma}}{N}\right)^{\cdot}-V_{, \sigma}=0
$$

Introducing the proper radial coordinate $\tau \equiv \int N \mathrm{~d} \xi$, the equations are rewritten as

$$
\begin{aligned}
& \frac{3}{a^{2}}\left(\frac{\mathrm{d} a}{\mathrm{~d} \tau}\right)^{2}-\frac{3 K}{a^{2}}=\frac{1}{2}\left(\frac{\mathrm{d} \sigma}{\mathrm{d} \tau}\right)^{2}-V(\sigma)-\Lambda_{ \pm}, \\
& \frac{\mathrm{d}^{2} \sigma}{\mathrm{d} \tau^{2}}+3\left(\frac{\mathrm{d} a}{\mathrm{~d} \tau}\right) \frac{\mathrm{d} \sigma}{\mathrm{d} \tau}-V_{, \sigma}(\sigma)=0 .
\end{aligned}
$$

As explained in the previous section, the tunneling rate (2.6) is given by the Euclidean action evaluated for a solution of Eqs. (3.18) and (3.19). In the following, we construct a HM solution and then evaluate the action for it.

\section{HAWKING-MOSS SOLUTION}

\section{A. Evaluation of the tunneling rate}

A HM solution can be found by setting the tunneling field to the local maximum value, $\sigma(\xi)=\sigma_{\mathrm{top}}$. Then the equation of motion (3.19) is trivially satisfied and the Euclidean Friedmann equation (3.18) reduces to

$$
\frac{3}{a^{2}}\left(\frac{\mathrm{d} a}{\mathrm{~d} \tau}\right)^{2}-\frac{3 K}{a^{2}}=-V\left(\sigma_{\mathrm{top}}\right)-\Lambda_{ \pm} \equiv-\Lambda_{ \pm, \mathrm{eff}} .
$$


Setting the boundary condition as $a_{\mathrm{HM}}\left(H_{\mathrm{HM}} \tau= \pm \pi / 2\right)=0$ and assuming $\Lambda_{ \pm, \text {eff }}>0$, the HM solution is obtained as

$$
a_{\mathrm{HM}}(\tau)=H_{\mathrm{HM}}^{-1} \sqrt{K} \cos \left(H_{\mathrm{HM}} \tau\right) .
$$

Here, we have introduced the Hubble parameter of the physical metric by

$$
H_{\mathrm{HM}} \equiv \sqrt{\frac{\Lambda_{ \pm, \mathrm{eff}}}{3}}=\sqrt{\frac{V\left(\sigma_{\mathrm{top}}\right)+\Lambda_{ \pm}}{3}} .
$$

Inserting Eq. (4.1) into the Euclidian action given by Eqs. (3.6) and (3.8), and using $N^{-1} \dot{f}=\mathrm{d} f / \mathrm{d} \tau$, the total action can be expressed as

$$
S_{E}\left[a_{\mathrm{HM}}, \sigma_{\mathrm{top}}\right]=\int \mathrm{d}^{3} x \sqrt{\Omega} \int_{-\pi / 2 H_{\mathrm{HM}}}^{\pi / 2 H_{\mathrm{HM}}} \mathrm{d} \tau a_{H M}^{3}\left(2 \Lambda_{ \pm, \mathrm{eff}}-\frac{6 K}{a_{\mathrm{HM}}^{2}}+m_{g}^{2} Y_{ \pm} \sqrt{-\left(\frac{\mathrm{d} f_{\mathrm{HM}}}{\mathrm{d} \tau}\right)^{2}}\right),
$$

where, for brevity, we have introduced the parameter $Y_{ \pm}$in terms of $X_{ \pm}$as

$$
Y_{ \pm} \equiv 3\left(1-X_{ \pm}\right)+3 \alpha_{3}\left(1-X_{ \pm}\right)^{2}+\alpha_{4}\left(1-X_{ \pm}\right)^{3} .
$$

We also need the solution for the Stückelberg field $f$ to evaluate the action. It is given in terms of $a_{\mathrm{HM}}$ as in Eq. (3.13),

$$
b_{\mathrm{HM}}=F^{-1} \sqrt{K} \cosh \left(F f_{\mathrm{HM}}\right)=X_{ \pm} a_{\mathrm{HM}} .
$$

This can be solved for $f$ as

$$
f_{\mathrm{HM}}(\tau)=\frac{1}{F} \ln \left[\alpha_{\mathrm{HM}} \cos \left(H_{\mathrm{HM}} \tau\right) \pm \sqrt{\alpha_{\mathrm{HM}}^{2} \cos ^{2}\left(H_{\mathrm{HM}} \tau\right)-1}\right],
$$

where the parameter $\alpha$ is defined as

$$
\alpha_{\mathrm{HM}} \equiv X_{ \pm} \frac{F}{H_{\mathrm{HM}}} .
$$

As clear from the above definition, the parameter $\alpha_{\mathrm{HM}}$ represents the ratio of the Hubble parameter of the fiducial metric and that of the physical metric. Since $X_{ \pm}$should be positive for the constraint (3.13) to be satisfied, $\alpha_{\mathrm{HM}}$ is also positive.

Finally, we evaluate the derivative of $f$ since the action (4.4) depends on the Stückelberg field only through $\mathrm{d} f / \mathrm{d} \tau$. Taking a derivative of Eq. (4.6) with respect to $\tau$, one obtains

$$
\frac{\mathrm{d} f_{\mathrm{HM}}}{\mathrm{d} \tau}=-\frac{X_{ \pm} \sin \left(H_{\mathrm{HM}} \tau\right)}{\sinh \left(F f_{H M}(\tau)\right)}
$$

where $\sinh \left(F f_{H M}\right)$ can be calculated from Eq. (4.6) as

$$
\sinh \left(F f_{H M}\right)= \pm \sqrt{\alpha_{\mathrm{HM}}^{2} \cos ^{2}\left(H_{\mathrm{HM}} \tau\right)-1} .
$$

Though we have obtained " \pm " branches for $\mathrm{d} f / \mathrm{d} \tau$ here, the sign is irrelevant because the action (4.4) depends only on its square,

$$
\left(\frac{\mathrm{d} f_{\mathrm{HM}}}{\mathrm{d} \tau}\right)^{2}=\frac{X_{ \pm}^{2} \sin ^{2}\left(H_{\mathrm{HM}} \tau\right)}{\alpha_{\mathrm{HM}}^{2} \cos ^{2}\left(H_{\mathrm{HM}} \tau\right)-1}
$$

Here we note that we can evaluate $\mathrm{d} f_{E} / \mathrm{d} \tau\left(f_{E}=-i f\right)$ in a similar way for the Minkowski fiducial metric by setting $b_{\mathrm{HM}}=\sqrt{K} f_{E}$ in Eq. (4.6). Taking a derivative with respect to $\tau$, one obtains

$$
\frac{\mathrm{d} f_{E, \mathrm{HM}}}{\mathrm{d} \tau}=-X_{ \pm} \sin \left(H_{\mathrm{HM}} \tau\right) .
$$


The square of this equation coincides with Eq. (4.11) with $\alpha_{\mathrm{HM}}=0$ after making the replacement $f_{E}=-i f$. Hence, the analysis below includes the results for the Minkowski fiducial metric.

Note that the solution for $\phi_{\mathrm{HM}}^{0}=f_{\mathrm{HM}}$ does not diverge in the limit $m_{g} \rightarrow 0$. Hence, our results continuously reduce to the results in GR. This is consistent with the observations that there is no vDVZ discontinuity in the self-accelerating branch [15, 22, 23].

It should also be noted that the induced metric $G_{a b} \partial_{\mu} \phi^{a} \partial_{\nu} \phi^{b}$ in the case of $\alpha_{\mathrm{HM}}>1$ has a "singularity", where $\dot{f}$ becomes infinite, and becomes neither pure imaginary nor pure real. The singularity would also appear even if we had started from the Euclidean signature for the fiducial metric. In this case, the four sphere for the fiducial metric would crunch before the physical one does. For this reason, we concentrate on the case where $\alpha_{\mathrm{HM}} \leq 1$.

As easily seen from the solution (4.7), the Stückelberg field becomes complex in general. However, as we will see, the action turns out to be real for $\alpha_{\mathrm{HM}} \leq 1$. We also note that if we start from the Euclidean fiducial metric, $f_{\mathrm{HM}}$ and $\mathrm{d} f_{\mathrm{HM}} / \mathrm{d} \tau$ become real for $\alpha_{\mathrm{HM}} \leq 1$.

\section{Action for the case $\alpha \leq 1$}

In the action (3.6), the only non-trivial term is the one that depends on $\mathrm{d} f / \mathrm{d} \tau$. Hence, we first evaluate this part. Substituting the solution (4.11), we find

$$
\begin{aligned}
m_{g}^{2} Y_{ \pm} \int_{-\pi / 2 H_{\mathrm{HM}}}^{\pi / 2 H_{\mathrm{HM}}} \mathrm{d} \tau a_{H M}^{3} \sqrt{-\left(\frac{\mathrm{d} f_{\mathrm{HM}}}{\mathrm{d} \tau}\right)^{2}} & =\frac{2 m_{g}^{2} Y_{ \pm} X_{ \pm}}{H_{\mathrm{HM}}^{4}} K^{\frac{3}{2}} \int_{0}^{\pi / 2} \mathrm{~d}\left(H_{\mathrm{HM}} \tau\right) \frac{\cos ^{3}\left(H_{\mathrm{HM}} \tau\right) \sin \left(H_{\mathrm{HM}} \tau\right)}{\sqrt{1-\alpha_{\mathrm{HM}}^{2} \cos ^{2}\left(H_{\mathrm{HM}} \tau\right)}} \\
& =\left.\frac{2 m_{g}^{2} Y_{ \pm} X_{ \pm}}{3 \alpha_{\mathrm{HM}}^{4} H_{\mathrm{HM}}^{4}} K^{\frac{3}{2}} \sqrt{1-z^{2}}\left(2+z^{2}\right)\right|_{\alpha_{\mathrm{HM}}} ^{0}, \quad\left(z \equiv \alpha_{\mathrm{HM}} \cos \left(H_{\mathrm{HM}} \tau\right)\right) \\
& =\frac{2 m_{g}^{2} Y_{ \pm} X_{ \pm}}{3 \alpha_{\mathrm{HM}}^{4} H_{\mathrm{HM}}^{4}} K^{\frac{3}{2}}\left[2-\sqrt{1-\alpha_{\mathrm{HM}}^{2}}\left(2+\alpha_{\mathrm{HM}}^{2}\right)\right]
\end{aligned}
$$

As claimed, the action is real.

\section{Hawking-Moss action}

From the result of the previous subsection, we obtain the Euclidean action as

$$
\begin{aligned}
S_{\mathrm{HM}} & \equiv S_{E}\left[a_{\mathrm{HM}}, \sigma_{\mathrm{top}}\right]=-\frac{8 \pi^{2}}{H_{\mathrm{HM}}^{2}}\left[1-\frac{Y_{ \pm} X_{ \pm}}{6 \alpha_{\mathrm{HM}}^{4}}\left(\frac{m_{g}}{H_{\mathrm{HM}}}\right)^{2}\left(2-\sqrt{1-\alpha_{\mathrm{HM}}^{2}}\left(2+\alpha_{\mathrm{HM}}^{2}\right)\right)\right] \\
& =-\frac{8 \pi^{2}}{H_{\mathrm{HM}}^{2}}\left[1-\frac{Y_{ \pm} X_{ \pm}}{6}\left(\frac{m_{g}}{H_{\mathrm{HM}}}\right)^{2} A\left(\alpha_{\mathrm{HM}}\right)\right] ; \quad A(\alpha) \equiv \frac{2-\sqrt{1-\alpha^{2}}\left(2+\alpha^{2}\right)}{\alpha^{4}},
\end{aligned}
$$

where $\int d^{3} x \sqrt{\Omega}=2 \pi^{2} K^{-3 / 2}$ has been used. As previously explained, we consider only the case $\alpha_{H M} \leq 1$ since a singularity would appear otherwise. In addition to the standard first term determined by the Hubble parameter $H_{\mathrm{HM}}$ (which nevertheless contains a contribution of the mass term $\Lambda_{ \pm}$), there apprears a mass-dependent term. The presence of this second term may be regarded as the genuine effect of the graviton mass. We also note here that the function $A(\alpha)$ is regular at $\alpha=0$, which corresponds to the case of the Minkowski fiducial metric.

As given by Eq. (2.6), the tunneling rate is determined by the difference between the HM action $S_{\mathrm{HM}}$ given by Eq. (4.14) and the false vacuum action $S_{\mathrm{F}}=S_{E}\left(\bar{g}_{\mu \nu, F}, \bar{\phi}_{F}\right)$. The latter is given similarly as Eq. (4.14) by replacing the Hubble parameter by

$$
H_{\mathrm{F}} \equiv \sqrt{\frac{V\left(\sigma_{F}\right)+\Lambda_{ \pm}}{3}}
$$

and $\alpha$ by its false vacuum value, $\alpha_{\mathrm{F}} \equiv X_{ \pm} F / H_{\mathrm{F}}$. We note that $\alpha_{\mathrm{F}}$ should not exceed unity either. Since the potential energy at the false vacuum is always smaller that at the maximum, $V\left(\sigma_{F}\right)<V\left(\sigma_{\text {top }}\right)$, we have

$$
\alpha_{\mathrm{F}}>\alpha_{\mathrm{HM}} \equiv \frac{X_{ \pm} F}{H_{\mathrm{HM}}}
$$


Hence the constraint $\alpha_{\mathrm{F}} \leq 1$ gives a tighter constraint on the potential energy than that from $\alpha_{\mathrm{HM}} \leq 1$. Conversely, if the false vacuum exists without a singularity in the Stückelberg field, a regular HM solution always exists.

To see the effect of the second term, let us compare the current result with the HM action in GR with the same value of the Hubble parameter, $B^{(\mathrm{GR})} \equiv 8 \pi^{2}\left(-H_{\mathrm{HM}}^{-2}+H_{\mathrm{F}}^{-2}\right)$. We have

$$
\Delta B \equiv B-B^{(\mathrm{GR})}=\frac{4 \pi^{2}}{3} Y_{ \pm} X_{ \pm}\left(\frac{A\left(\alpha_{\mathrm{HM}}\right)}{H_{\mathrm{HM}}^{4}}-\frac{A\left(\alpha_{\mathrm{F}}\right)}{H_{\mathrm{F}}^{4}}\right) m_{g}^{2} .
$$

Note that the function $A(\alpha)$ is positive and a monotonically increasing function of $\alpha$ for $0<\alpha \leq 1$. Hence $0<A\left(\alpha_{\mathrm{HM}}\right)<A\left(\alpha_{\mathrm{F}}\right)$. Since $H_{\mathrm{HM}}^{-1}<H_{\mathrm{F}}^{-1}$, this means the sign of $\Delta B$ is determined by the sign of $Y_{ \pm}$. Namely, for $Y_{ \pm}>0(<0)$, we have $\Delta B<0(>0)$, and hence the tunneling rate is enhanced (suppressed) relative to the case of GR.

\section{B. Parameter dependence of the tunneling rate}

In the above, we have seen the effect of the graviton mass on the tunneling rate is determined by the sign of $Y_{ \pm}$. In this subsetion, we analyze the dependence of the tunneling rate on the parameters $\alpha_{3}$ and $\alpha_{4}$ by examining the sign of $Y_{ \pm}$.

\section{General case with $m_{g} \neq 0$}

As shown in the previous subsection, the change in the tunneling rate is determined by the sign of $Y_{ \pm}$, which is given in terms of $X_{ \pm}$as given by Eq. (4.5). Let us recapitulate it here:

$$
\begin{aligned}
& X_{ \pm}=\frac{1+2 \alpha_{3}+\alpha_{4} \pm \sqrt{1+\alpha_{3}+\alpha_{3}^{2}-\alpha_{4}}}{\alpha_{3}+\alpha_{4}}, \\
& Y_{ \pm}=3\left(1-X_{ \pm}\right)+3 \alpha_{3}\left(1-X_{ \pm}\right)^{2}+\alpha_{4}\left(1-X_{ \pm}\right)^{3} .
\end{aligned}
$$

In Fig. 2, we show the sign of $Y_{ \pm}$in the parameter space $\left(\alpha_{3}, \alpha_{4}\right)$ for Branch $\mathrm{II}_{+}$(left panel) and Branch $\mathrm{II}_{-}$ (right panel) solutions. As seen from the figure, the tunneling rate is enhanced (suppressed) for Branch $\mathrm{II}_{+}$ (Branch II_) in a large region of the parameter space. It should be noted again that $\alpha_{F} \leq 1$ gives rise to a constraint on $V\left(\sigma_{\mathrm{F}}\right)$.

Next, we focus on some limiting cases and analytically investigate the parameter dependence of the tunneling rate.

$$
\text { 2. } \alpha_{3}=0
$$

In this case, from Eq. (4.18)

$$
X_{ \pm}=\frac{1}{\alpha_{4}}\left(1+\alpha_{4} \pm \sqrt{1-\alpha_{4}}\right), \quad Y_{ \pm}=\left(1-X_{ \pm}\right)\left(2+\alpha_{4}\left(1-X_{ \pm}\right)^{2}\right) ，
$$

and from Eq. (3.16),

$$
\Lambda_{ \pm}=-\frac{m_{g}^{2}}{\alpha_{4}^{2}}\left[2-3 \alpha_{4} \pm 2\left(1-\alpha_{4}\right)^{\frac{3}{2}}\right] .
$$

Note that the parameter $\alpha_{4}$ should satisfy $\alpha_{4} \leq 1$ for $X_{ \pm}$to be real. In the following, we evaluate the HM action for Branch $\mathrm{II}_{+}$and Branch II- separately.

- Branch $\mathrm{II}_{+}$

In this branch, we have

$$
X_{+}=\frac{1}{\alpha_{4}}\left(1+\alpha_{4}+\sqrt{1-\alpha_{4}}\right), \quad Y_{+}=-\frac{2}{\alpha_{4}^{2}}\left(1+\sqrt{1-\alpha_{4}}\right)\left(1+\alpha_{4}+\sqrt{1-\alpha_{4}}\right),
$$



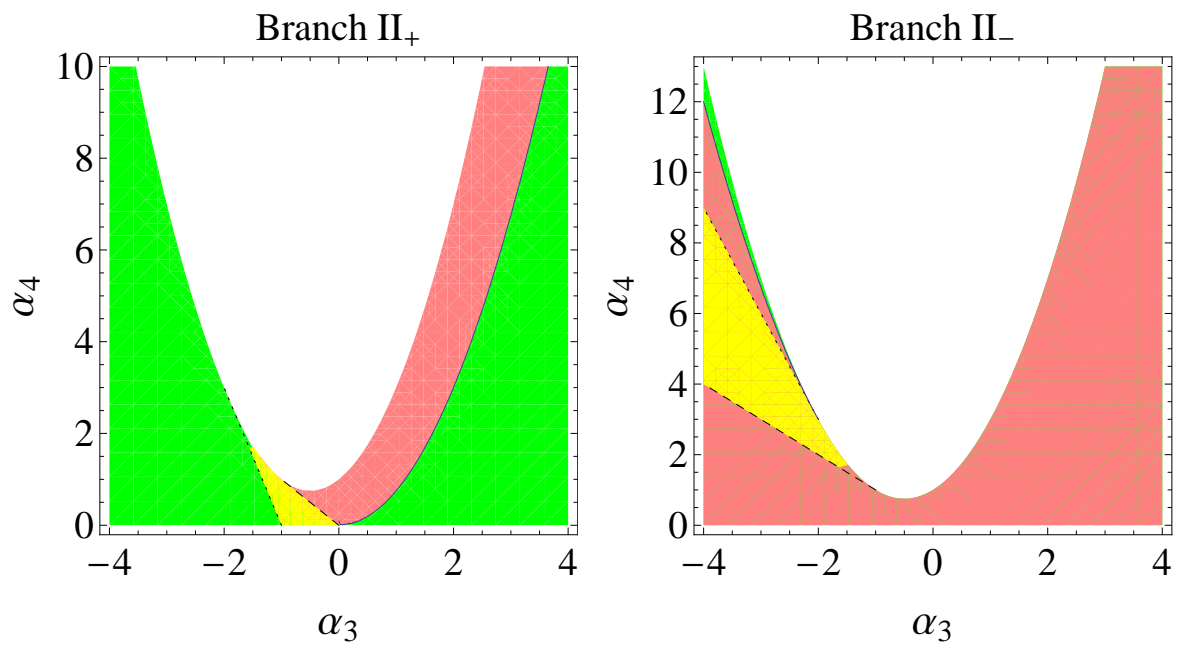

FIG. 2: The sign of $Y_{ \pm}$is shown for Branch $\mathrm{II}_{+}$(left panel) and Branch $\mathrm{II}_{-}$(right panel) solutions. The white and yellow regions corresponds to $1+\alpha_{3}+\alpha_{3}^{2}-\alpha_{4}<0$ and $X_{ \pm}<0$ respectively, which should be excluded since the cosmological solution does not exist in these regions [12, 22]. The green regions correspond to $Y_{ \pm}>0$, which implies that the HM tunneling rate is enhanced in comparison to the GR case for the same Hubble parameter $H_{\mathrm{HM}}$, while the pink regions correspond to $Y_{ \pm}<0$, which implies that the HM tunneling rate is suppressed. Along the solid lines (defining the boundary between green and pink regions), $X_{ \pm}=1$ and hence $Y_{ \pm}=0$. Hence the HM solution (4.14) reduces to that in GR. The dotted lines denote $X_{ \pm}=0$ where the solution ceases to exist. Along the dashed lines, $X_{ \pm}$diverges and thus defines another boundary of the solution space.

and

$$
\Lambda_{+}=-\frac{m_{g}^{2}}{\alpha_{4}^{2}}\left[2-3 \alpha_{4}+2\left(1-\alpha_{4}\right)^{\frac{3}{2}}\right] .
$$

From the expression above, one immediately finds that $\alpha_{4}=0$ can be a singular point for Branch $\mathrm{II}_{+}$. To study this case, we set $\alpha_{3}=\alpha_{4}=\epsilon \ll 1$ and take the limit $\epsilon \rightarrow 0$. Then one obtains

$$
\Lambda_{+}\left(\alpha_{3}=\alpha_{4}=0\right)=-\frac{m_{g}^{2}}{2 \epsilon^{2}}\left[2+\frac{3 \epsilon^{2}}{2}+\mathcal{O}\left(\epsilon^{3}\right)\right],
$$

which leads to

$$
\lim _{\epsilon \rightarrow 0} \Lambda_{+}\left(\alpha_{3}=\alpha_{4}=0\right)=-\infty .
$$

So $\alpha_{3}=\alpha_{4}=0$ is singular in this branch, which should be excluded in the analysis. From the condition $\alpha_{\mathrm{F}} \leq 1$, we obtain the constraint on $V\left(\sigma_{\mathrm{F}}\right)$ by using Eqs. (4.8) and (4.21):

$$
V\left(\sigma_{\mathrm{F}}\right) \geq \frac{\left(2 \gamma_{4}^{3}+3 \gamma_{4}^{2}-1\right) m_{g}^{2}+3\left(\gamma_{4}^{2}-\gamma_{4}-2\right)^{2} F^{2}}{\left(1-\gamma_{4}^{2}\right)^{2}}=\frac{\left(2 \gamma_{4}-1\right) m_{g}^{2}+3\left(\gamma_{4}-2\right)^{2} F^{2}}{\left(\gamma_{4}-1\right)^{2}},
$$

where

$$
\gamma_{4} \equiv \sqrt{1-\alpha_{4}}(\geq 0)
$$

An interesting consequence of this constraint is the absence of a (meta-)stable vacuum state below this bound. For example, even if there is a potential minimum below this bound, tunneling to such a state may be prohibited.

In terms of $\gamma_{4}$, Eqs. (4.21) can be re-expressed as

$$
X_{+}=\frac{\gamma_{4}-2}{\gamma_{4}-1}, \quad Y_{+}=2 \frac{\gamma_{4}-2}{\left(\gamma_{4}-1\right)^{2}} .
$$

Thus the condition $X_{+}>0$ requires either $\gamma_{4}>2\left(\alpha_{4}<-3\right)$ for which we have $Y_{+}>0$, or $0 \leq \gamma_{4}<1$ $\left(1 \geq \alpha_{4}>0\right)$ for which $Y_{+}<0$. Therefore the tunneling rate is enhanced for $\alpha_{4}<-3$ and suppressed for $0<\alpha_{4} \leq 1$. The range $-3<\alpha_{4}<0$ is excluded because a solution ceases to exist there. 
In order for the solution to be a four-sphere, the effective cosmological constant must be positive definite, $\Lambda_{+, \mathrm{eff}}>0$. We find this is always satisfied, since

$$
\Lambda_{+, \mathrm{eff}} \geq V\left(\sigma_{\mathrm{F}}\right)+\Lambda_{+} \geq 3 F^{2} X_{ \pm}^{2}>0 .
$$

We again note that $\gamma_{4}=1\left(\alpha_{4}=0\right)$ is a singular point, which coincides with the conclusion that Branch $\mathrm{II}_{+}$ does not exist for $\alpha_{3}=\alpha_{4}=0$.

- Branch II_

In this branch, we have

$$
\begin{aligned}
& X_{-}=\frac{1}{\alpha_{4}}\left(1+\alpha_{4}-\sqrt{1-\alpha_{4}}\right)=\frac{\gamma_{4}+2}{\gamma_{4}+1}, \\
& Y_{-}=\frac{2}{\alpha_{4}^{2}}\left(-2+2 \sqrt{1-\alpha_{4}}+\alpha_{4} \sqrt{1-\alpha_{4}}\right)=-2 \frac{\gamma_{4}+2}{\left(\gamma_{4}+1\right)^{2}},
\end{aligned}
$$

and

$$
\Lambda_{-}=-\frac{m_{g}^{2}}{\alpha_{4}^{2}}\left[2-3 \alpha_{4}-2\left(1-\alpha_{4}\right)^{\frac{3}{2}}\right]=\frac{2 \gamma_{4}+1}{\left(\gamma_{4}+1\right)^{2}} m_{g}^{2},
$$

where, as before, $\gamma_{4}=\sqrt{1-\alpha_{4}}$ and the parameter $\alpha_{4}$ should not exceed unity. We note that in contrast with Branch $\mathrm{II}_{+}$, here $\alpha_{3}=\alpha_{4}=0$ is regular, which implies a finite $\Lambda_{+}\left(\alpha_{3}=\alpha_{4}=0\right)=3 m_{g}^{2} / 4$.

Similar to the case of Branch $\mathrm{II}_{+}$, we find a constraint on $V\left(\sigma_{F}\right)$ on Branch $\mathrm{II}_{-}$from the condition $\alpha_{4} \leq 1$ as

$$
V\left(\sigma_{F}\right) \geq \frac{\left(-2 \gamma_{4}^{3}+3 \gamma_{4}^{2}-1\right) m_{g}^{2}+3\left(\gamma_{4}^{2}+\gamma_{4}-2\right)^{2} F^{2}}{\left(1-\gamma_{4}^{2}\right)^{2}}=\frac{-\left(2 \gamma_{4}+1\right) m_{g}^{2}+3\left(\gamma_{4}+2\right)^{2} F^{2}}{\left(\gamma_{4}+1\right)^{2}} .
$$

It is clear that the limit $\gamma_{4} \rightarrow 1\left(\alpha_{4} \rightarrow 0\right)$ is regular in this case of Branch $\mathrm{II}_{-}$, which is consistent with the case $\alpha_{3}=\alpha_{4}=0$ studied previously. We note that there is no restriction on the range of $\alpha_{4}$ except for the condition that $\alpha_{4} \leq 1$.

From Eqs. (4.29), we see that $Y_{-}$is always negative. Therefore, the tunneling rate is always suppressed in this case.

\section{Vanishing $\mathcal{L}_{2}$ term}

In this subsection, we consider a special case when $\mathcal{L}_{2}$ vanishes but $\mathcal{L}_{3}$ and $\mathcal{L}_{4}$ are non-vanishing. To achieve this, we take the limit $m_{g}^{2} \rightarrow 0$, while keeping $A_{3} \equiv m_{g}^{2} \alpha_{3}$ and $A_{4} \equiv m_{g}^{2} \alpha_{4}$ finite. Substituting $A_{3}$ and $A_{4}$ for $\alpha_{3}$ and $\alpha_{4}$ in Eq. (3.16) and taking $m_{g}^{2} \rightarrow 0, \Lambda_{ \pm}$is calculated as

$$
\Lambda_{ \pm}=-\frac{2 A_{3}^{3}}{\left(A_{3}+A_{4}\right)^{2}}\left[1 \pm \operatorname{sgn}\left(\mathrm{A}_{3}\right)\right]
$$

where $\operatorname{sgn}\left(\mathrm{A}_{3}\right) \equiv\left|\mathrm{A}_{3}\right| / \mathrm{A}_{3}$ denotes the sign of the parameter $A_{3}$. The sign function appears due to the presence of the square root in Eq. (3.16). Similarly, from Eq. (3.13), $X_{ \pm}$can be obtained as

$$
X_{ \pm}=1+\frac{A_{3}}{A_{3}+A_{4}}\left[1 \pm \operatorname{sgn}\left(\mathrm{A}_{3}\right)\right] .
$$

Without loss of generality, we assume $A_{3}>0$ since the flip of sign only exchanges the roles of Branch $\mathrm{II}_{+}$and Branch II_.

- Branch $\mathrm{II}_{+}$

In this branch, from Eqs. (4.32) and 4.33), we have

$$
\Lambda_{+}=-\frac{4 A_{3}^{3}}{\left(A_{3}+A_{4}\right)^{2}}<0, \quad X_{+}=1+\frac{2 A_{3}}{A_{3}+A_{4}},
$$


and

$$
\begin{aligned}
m_{g}^{2} Y_{+} & =3 A_{3}\left(1-X_{+}\right)^{2}+A_{4}\left(1-X_{+}\right)^{3} \\
& =\left(1-X_{+}\right)^{2} X_{+} A_{3} .
\end{aligned}
$$

Thus $m_{g}^{2} Y_{-}$is always positive provided $X_{-}>0$. From Eq. (4.34), this is guaranteed if the parameter $A_{4}$ is either in the range $A_{4}<-3 A_{3}$ or $-A_{3}<A_{4}$. The tunneling rate is always enhanced in this case.

Next let us consider the condition $\alpha_{\mathrm{F}}<1$. From Eq. (4.34), we find

$$
\alpha_{\mathrm{F}}^{2}=\frac{3 F^{2}\left(3 A_{3}+A_{4}\right)^{2}}{\left(A_{3}+A_{4}\right)^{2} V\left(\sigma_{\mathrm{F}}\right)-4 A_{3}^{3}} \leq 1 .
$$

Hence the constraint on $V\left(\sigma_{\mathrm{F}}\right)$ is found as

$$
V\left(\sigma_{\mathrm{F}}\right) \geq \frac{3 F^{2}\left(3 A_{3}+A_{4}\right)^{2}+4 A_{3}^{3}}{\left(A_{3}+A_{4}\right)^{2}} .
$$

Again the positivity of the effective cosmological constant, $\Lambda_{+, \text {eff }}>0$, is guaranteed because

$$
\Lambda_{+, \text {eff }} \geq V\left(\sigma_{\mathrm{F}}\right)+\Lambda_{+} \geq 3 F^{2} X_{+}^{2}>0 .
$$

- Branch II

In this branch, from Eqs. (4.32) and (4.33), we find

$$
\Lambda_{-}=0, \quad X_{-}=1, \quad m_{g}^{2} Y_{-}=0 .
$$

This implies that the action in this case reduces to that of GR. There is no difference from GR in this branch.

From Eq. (4.39), the condition $\alpha_{\mathrm{F}} \leq 1$ is expressed as

$$
\alpha_{\mathrm{F}}^{2}=\frac{3 F^{2}}{V\left(\sigma_{\mathrm{F}}\right)} \leq 1,
$$

which leads to the constraint on $V\left(\sigma_{\mathrm{F}}\right)$ as

$$
V\left(\sigma_{\mathrm{F}}\right) \geq 3 F^{2} .
$$

\section{SUMMARY AND DISCUSSION}

In this paper, we investigated the stability of a vacuum in the landscape of vacua in a theory of non-linear massive gravity. For this purpose, we derived the Hawking-Moss (HM) solution in a simple case where a tunneling scalar field is minimally coupled with gravity and evaluated its Euclidean action. We obtained three branches of the solution, which we labeled as Branch $\mathrm{I}$ and Branch $\mathrm{II}_{ \pm}$, where Branch $\mathrm{II}_{ \pm}$corresponds to the self-accelerating branch in Ref. 12, 22]. We analyzed the contribution of the graviton mass terms to the Euclidean action, hence to the tunneling rate. We focused on Branch- $\mathrm{II}_{ \pm} \mathrm{HM}$ solutions because a Branch-I HM solution is shown to be equivalent to a Branch-II solution with a different set of the model parameters.

The Euclidean action of the HM instanton is found to have two distinct terms. In addition to the standard contribution determined by the Hubble parameter of the HM solution, we obtained a mass-dependent nonstandard term. To study the effect of this latter non-standard contributions, we compared the tunneling rate to that in GR for the same value of the Hubble parameter.

For Branch $\mathrm{II}_{ \pm}$, the enhancement or suppression of the tunneling rate relative to GR is determined by the sign of a quantity, denoted by $Y_{ \pm}$, and it depends on the model parameters $\alpha_{3}$ and $\alpha_{4}$. We found that in a wide area of the parameter space the tunneling rate is enhanced for Branch $\mathrm{II}_{+}$and suppressed for Branch $\mathrm{II}_{-}$.

We also found that the solution can exist only if the Hubble parameter of the ratio of the physical metric to the fiducial one is greater than $X_{ \pm}$. As a result, the form of the potential is constrained. This seems to imply that tunneling from or to a vacuum whose energy density is less than a critical value is prohibited. This result is in sharp contrast to GR for which there is no bound on the minimum value of the potential. 
As tunneling mediated by a HM instanton is considered to be equivalent to stochastic process of going over the barrier due to large vacuum fluctuations intrinsic to light scalar fields in de Sitter space [42], it is interesting to understand the role of the graviton mass terms in the context of stochastic dynamics.

We found that the tunneling rate can be enhanced or suppressed depending on the model parameters for a simple case of a tunneling field minimally couples with gravity. Though we discussed this simple case as a first step, it will be interesting to see how the tunneling process proceeds when the model parameters such as the graviton mass depend on the tunneling field. In this case, the effective cosmological constant of a vacuum can be larger than the other while its potential energy is smaller because of the tunneling field dependence of the graviton mass.

Finally, let us comment on the limitation of our analysis. Since massive gravity exhibits the strong coupling at high energy scales, it requires UV completion above its cutoff scale. Namely, our analysis cannot apply to an arbitrarily small size instanton nor to the potential of an arbitrarily high energy scale, though the limitation depends on how massive gravity is UV completed. The cutoff scale of massive gravity is determined by the graviton mass and it is very low if the graviton mass is of the order of the present Hubble parameter to explain the current accelerated expansion of the universe. However, as mentioned above, the graviton mass may depend on the tunneling field and may have a larger value in the early universe. In this case, our analysis can be applied to the tunneling process at high energy scales.

\section{Appendix A: HM solution for Branch I}

In the text, we focused on HM solutions based on Branch II constraint (3.9). Here, we show that the HM solution in Branch I is equivalent to the one in Branch II with a different set of the model parameters.

Inserting Eq. (2.10) into Eq. (3.11), we obtain

$$
F^{2} b^{2}+a^{\prime 2}=K
$$

Hence

$$
b=\frac{\sqrt{K-a^{\prime 2}}}{F} .
$$

Inserting this into Eq. (3.14) and setting $\sigma \equiv \sigma_{\text {top }}$, we obtain an algebraic equation,

$$
3 F^{2} \theta^{2}-V\left(\sigma_{\text {top }}\right)-\rho_{g}=0,
$$

where

$$
\rho_{g} \equiv-m_{g}^{2}(1-\theta)\left[3(2-\theta)+\alpha_{3}(1-\theta)(4-\theta)+\alpha_{4}(1-\theta)^{2}\right], \quad \theta \equiv \frac{\sqrt{K-a^{\prime 2}}}{F a}=\frac{b}{a} .
$$

Hence, $\theta=b / a$ becomes constant. The HM solution in Branch I is obtained as

$$
a_{\mathrm{HM}}^{I}(\tau)=(\theta F)^{-1} \sqrt{K} \cos (\theta F \tau) .
$$

Comparing this with Eqs. (3.13) and (4.2), we see that the form of the solutions are the same except that $X_{ \pm}$ and $H_{\mathrm{HM}}$ are replaced by $\theta$ and $\theta F$, respectively. We note that the quantity corresponding to $\alpha \equiv X_{ \pm} F / H_{\mathrm{HM}}$ in Branch I is unity.

Thus, we conclude that the analysis of the HM solutions for Branch II can be directly applied to those for Branch I if we make the replacement $X_{ \pm} \rightarrow \theta$ and $H_{\mathrm{HM}} \rightarrow \theta F$. Note that $\theta$ depends on $F$, $m_{g}$, and $V\left(\sigma_{\text {top }}\right)$, as well as $\alpha_{3}$ and $\alpha_{4}$. Hence, the dependence on the model parameters becomes more complicated in Branch I than in Branch II.

\section{Appendix B: List of symbols}

Throughout the paper, there appear many parameters which are denoted by various symbols. For convenience, we list some of them who play important roles in the table — 
TABLE I: List of symbols

\begin{tabular}{crr}
\hline Symbols & Definition & First appearance \\
\hline$X_{ \pm}$ & See Eq. $\sqrt{3.13}$ & \\
$\Lambda_{ \pm}$ & See Eq. 3.16$)$ & \\
$\Lambda_{ \pm, \text {eff }}$ & $V\left(\sigma_{\text {top }}\right)+\Lambda_{ \pm}$ & \\
$Y_{ \pm}$ & See Eq. (4.5) & \\
$H_{\mathrm{HM}}$ & $\sqrt{\Lambda_{ \pm, \text {eff }} / 3}$ & \\
$\alpha_{\mathrm{HM}}$ & $F X_{ \pm} / H_{\mathrm{HM}}$ & \\
$H_{\mathrm{F}}$ & $\sqrt{\left(V\left(\sigma_{F}\right)+\Lambda_{ \pm}\right) / 3}$ & \\
$\alpha_{F}$ & $F X_{ \pm} / H_{\mathrm{F}}$ & Below \\
\hline
\end{tabular}

\section{Acknowledgments}

We thank C. de Rham, A. J. Tolley, R. Gobbetti, A. E. Gümrükçüoğlu, C. Lin, S. Mukohyama, K. Sugimura and T. Tanaka for helpful discussions. This work was supported in part by the Grant-in-Aid for the Global COE Program "The Next Generation of Physics, Spun from Universality and Emergence" from the Ministry of Education, Culture, Sports, Science and Technology (MEXT) of Japan, and by JSPS Grant-in-Aid for Scientific Research (A) No. 21244033. RS is supported by a JSPS Grant-in-Aid through the JSPS postdoctoral fellowship. We acknowledge the workshop YITP-W-11-26 at Yukawa Institute for Theoretical Physics, Kyoto University, where this project was initiated, and we are grateful to the participants of the workshop YITP-T-12-04 for valuable discussions and comments.

[1] M. Fierz and W. Pauli, Proc. Roy. Soc. Lond. A 173, 211-232 (1939).

[2] D. G. Boulware and S. Deser, Phys. Rev. D 6, 3368-3382 (1972).

[3] P. Creminelli, A. Nicolis, M. Papucci and E. Trincherini, JHEP 0509, 003 (2005). [hep-th/0505147].

[4] V. A. Rubakov and P. G. Tinyakov, Phys. Usp. 51, 759-792 (2008). [arXiv:0802.4379 [hep-th]].

[5] K. Hinterbichler, Rev. Mod. Phys. 84, 671-710 (2012). [arXiv:1105.3735 [hep-th]].

[6] C. de Rham and G. Gabadadze, Phys. Rev. D 82, 044020 (2010). [arXiv:1007.0443 [hep-th]].

[7] C. de Rham, G. Gabadadze and A. J. Tolley, Phys. Rev. Lett. 106, 231101 (2011). [arXiv:1011.1232 [hep-th]].

[8] S. F. Hassan and R. A. Rosen, JHEP 1107, 009 (2011). [arXiv:1103.6055 [hep-th]].

[9] S. F. Hassan and R. A. Rosen, Phys. Rev. Lett. 108, 041101 (2012). [arXiv:1106.3344 [hep-th]].

[10] S. F. Hassan, R. A. Rosen and A. Schmidt-May, JHEP 1202, 026 (2012) [arXiv:1109.3230 [hep-th]].

[11] G. D'Amico, C. de Rham, S. Dubovsky, G. Gabadadze, D. Pirtskhalava and A. J. Tolley, Phys. Rev. D 84, 124046 (2011). [arXiv:1108.5231 [hep-th]].

[12] A. E. Gümrükçüoğlu, C. Lin and S. Mukohyama, JCAP 11, 030 (2011). [arXiv:1109.3845 [hep-th]].

[13] T. Kobayashi, M. Siino, M. Yamaguchi and D. Yoshida, Phys. Rev. D 86, 061505 (2012). [arXiv:1205.4938 [hep-th]].

[14] P. Gratia, W. Hu and M. Wyman, Phys. Rev. D 86, 061504(2012). [arXiv:1205.4241 [hep-th]].

[15] K. Koyama, G. Niz and G. Tasinato, Phys. Rev. D 84, 064033 (2011). [arXiv:1104.2143 [hep-th]].

[16] K. Koyama, G. Niz and G. Tasinato, Phys. Rev. Lett. 107, 131101 (2011). [arXiv:1103.4708 [hep-th]].

[17] D. Langlois and A. Naruko, Class. Quant. Grav. 29, 202001 (2012) [arXiv:1206.6810 [hep-th]].

[18] H. Motohashi and T. Suyama, Phys. Rev. D 86, 081502 (2012) [arXiv:1208.3019 [hep-th]].

[19] A. Higuchi, Nucl. Phys. B 282, 397 (1987).

[20] L. Grisa and L. Sorbo, Phys. Lett. B 686, 273 (2010). [arXiv:0905.3391 [hep-th]].

[21] M. Fasiello and A. J. Tolley, JCAP 1211, 035 (2012). [arXiv:1206.3852 [hep-th]].

[22] A. E. Gümrükçüoğlu, C. Lin and S. Mukohyama, JCAP 1203, 006 (2012). [arXiv:1111.4107 [hep-th]].

[23] K. Koyama, G. Niz and G. Tasinato, JHEP 1112, 065 (2011). [arXiv:1110.2618 [hep-th]].

[24] A. De Felice, A. E. Gümrükçüoğlu and S. Mukohyama, Phys. Rev. Lett. 109, 171101(2012) [arXiv:1206.2080 [hepth]].

[25] G. Tasinato, K. Koyama and G. Niz, arXiv:1210.3627 [hep-th].

[26] S. Weinberg, Rev. Mod. Phys. 61, 1 (1989).

[27] S. Nobbenhuis, Found. Phys. 36, 613 (2006). [gr-qc/0411093].

[28] L. Susskind, In *Carr, Bernard (ed.): Universe or multiverse?* 247-266 [hep-th/0302219].

[29] Q. Huang, Y. Piao and S. Zhou, Phys. Rev. D 86, 124014 (2012). [arXiv:1206.5678 [hep-th]].

[30] E. N. Saridakis, arXiv:1207.1800 [gr-qc].

[31] C. Cai, C. Gao and E. N. Saridakis, JCAP 1210, 048 (2012) [arXiv:1207.3786 [astro-ph]].

[32] S. W. Hawking and I. G. Moss, Phys. Lett. B 110, 35 (1982). 
[33] S. R. Coleman and F. De Luccia, Phys. Rev. D 21, 3305 (1980).

[34] T. Tanaka and M. Sasaki, Prog. Theor. Phys. 88, 503 (1992)

[35] S. R. Coleman, V. Glaser and A. Martin, Commun. Math. Phys. 58, 211 (1978).

[36] S. F. Hassan and R. A. Rosen, JHEP 1202, 126 (2012) [arXiv:1109.3515 [hep-th]].

[37] M. S. Volkov, JHEP 1201, 035 (2012) [arXiv:1110.6153 [hep-th]].

[38] D. Comelli, M. Crisostomi, F. Nesti and L. Pilo, JHEP 1203, 067 (2012) [Erratum-ibid. 1206, 020 (2012)] [arXiv:1111.1983 [hep-th]].

[39] J. B. Hartle and S. W. Hawking, Phys. Rev. D 28, 2960 (1983).

[40] A. Vilenkin, Phys. Rev. D 30, 509 (1984).

[41] S. W. Hawking, Phys. Lett. B 134, 403 (1984).

[42] A. Starobinsky, In *De Vega, H.j. ( Ed.), Sanchez, N. ( Ed.): Field Theory, Quantum Gravity and Strings*, 107-126 